\title{
UWB at SemEval-2016 Task 7: Novel Method for Automatic Sentiment Intensity Determination
}

\author{
Ladislav Lenc, Pavel Král and Václav Rajtmajer \\ Dept. of Computer Science \& Engineering \\ Faculty of Applied Sciences \\ University of West Bohemia \\ Plzeň, Czech Republic \\ $\{l$ lenc, pkral, rajtmajv\}@kiv.zcu.cz
}

\begin{abstract}
We present a novel method for determining sentiment intensity. The main goal is to assign a phrase a score from 0 to 1 which indicates the strength of its association with positive sentiment. The proposed model uses a rich set of features with Gaussian processes regression model that computes the final score. The system was evaluated on the data from 7th task of SemEval 2016. Our regression model trained on the development data reached Kendall rank correlation of 0.659 on general English phrases and 0.414 on English Twitter test data.
\end{abstract}

\section{Introduction}

A great part of today's communication takes place on the Internet. Many companies make their business on the Web, it is possible to read newspapers through this media, etc. All these topics are tightly connected with forums, reviews and comments where users express their opinions and feelings. This great amount of short messages is a very rich source of information. For example companies can survey how people appreciate their goods or services. Social media such as Twitter or Facebook can also provide large amount of data for opinion and sentiment analysis.

Sentiment analysis can be seen as a part of opinion mining that gives the affective part of an opinion (Kim and Hovy, 2004). The short texts are usually assigned either positive or negative sentiment (i.e. sentiment polarity). It can be done for example by text categorization techniques. The minimum cuts are used for this task in (Pang and Lee, 2004). Another sentiment analysis approach based on Latent Dirichlet Allocation (LDA) is proposed in ( $\mathrm{Li}$ et al., 2010).

Many researchers evaluate their approaches on movie or product reviews. Lately, with the boom of social networks, there are also applications that analyze sentiment on Twitter (Wang et al., 2011).

An important progress was made thanks to the SemEval workshop (Rosenthal et al., 2015) in a sentiment analysis task. The task of determining sentiment intensity was introduced as a part of SemEval 2015 task 10. The goal of this task is to associate a word (or a short phrase) with a score that indicates its sentiment level. A score of 1 means that the phrase is fully positive whereas 0 is assigned to negative ones. This approach allows much more fine-grained sentiment evaluation. This year, sentiment intensity determination is the objective of the task 7 (Kiritchenko et al., 2016) and aims at three separate datasets: General English phrases, English Twitter and Arabic Twitter.

An interesting method that uses no linguistic resources and is based on word embeddings was proposed in (Astudillo et al., 2015). The system reached Kendall rank correlation of 0.625 on the SemEval 2015 test data. Another successful system (Zhang et al., 2015) used sentiment lexicons and a regression model to determine the sentiment intensity.

The proposed system computes a rich set of features which are further used to train a regression model that computes the final sentiment intensity score. Four different types of features are proposed:

- lexicon based features; 


\begin{tabular}{|l|l|l|l|l||l|l|l|}
\hline List & $\mathrm{hp}$ & $\mathrm{p}$ & $\mathrm{n}$ & $\mathrm{hn}$ & int & inv & dim \\
\hline Words & 171 & 772 & 782 & 554 & 83 & 15 & 31 \\
\hline
\end{tabular}

Table 1: Numbers of words in the JRC sentiment lexicon

- word2vec embeddings (Mikolov et al., 2013) based features;

- classifier based features;

- other features.

The paper is organized as follows. Section 2 describes the proposed system. Section 3 summarizes the experiments conducted with individual features and presents further the final results on the test data. Section 4 then concludes the paper and lists some perspectives for the future research.

\section{Proposed System}

The first part of this section is focused on the proposed features while the second one deals with the regression model.

The first group of the features used in the proposed system is built upon the sentiment lexicons.

\subsection{Sentiment Lexicons}

\subsubsection{AFINN}

AFINN 111 (Nielsen, 2011) contains 2,477 English words or 2-word phrases. All entries are associated with an integer value in the interval from -5 to 5.

Therefore, we have normalized these numbers to the interval $[0 ; 1]$.

\subsubsection{JRC}

This lexicon was created by using the approach proposed in (Steinberger et al., 2012). It contains four lists of words grouped according to their sentiment ( $h p$ - highly positive, $p=$ positive, $n=$ negative, $h n=$ highly negative) and additional lists of intensifiers (int), inverters (inv) and diminishers (dim). Table 1 summarizes the numbers of words in these lists.

We have joined the four lists and associated all entries with vales $0,0.25,0.75$ or 1 corresponding to its presence in the $h n, n, p$ or $h p$ list respectively.

\subsubsection{SentiWordNet}

SentiWordNet (SWN) is a large lexicon for sentiment analysis and opinion mining applica- tions (Esuli and Sebastiani, 2006). We used the version 3.0 (Baccianella et al., 2010) which contains the results of automatic annotation of synsets from the WordNet dictionary. Each synset is annotated with three scores: positivity, negativity and objectivity. The sum of these three values is always equal to 1. Each word can have several entries for different meanings and for different Parts Of Speech (POS) tags.

To obtain one sentiment intensity value, we compute a score according to the Equation 1.

$$
\frac{1}{P} \sum_{p=1}^{P} \sum_{m=1}^{M} \frac{1}{m}\left(\operatorname{Pos}_{p, m}-N e g_{p, m}\right)
$$

where $P$ is the number of POS tags that the word has entries for and $M$ is the number of meanings that the word can have. Posp, $m$ and Negp, $m$ are the positivity and negativity word values with the given POS $p$ and meaning $m$. The objectivity value was not used in this computation.

\subsection{Features}

We list below the features that we used to build our regression model. The features that are built upon the dictionaries (i.e. Direct search, W2V search and Rule based W2V search) are computed for each of the above described dictionaries.

1. Direct search: We search an intensity value of a particular word in the dictionary (if present, the result is its value, if not, the result is the neutral value 0.5 ). For multi-word phrases, we compute the arithmetic mean of all word values in the phrase.

2. W2V search: This approach is motivated by the assumption that semantically close words have often also similar sentiment (e.g. "nice" and "fine"). Of course, some exceptions can occur (e.g. "good" and "bad"), however we would like to minimize their impact by averaging a significant number of other close words.

Word2vec is used to identify $N$ semantically closest words from the dictionary for a word given. The resulting value is computed accord- 
ing to the Equation 2.

$$
\text { score }=\frac{1}{K N} \sum_{k=1}^{K} \sum_{n=1}^{N} \operatorname{int}\left(\operatorname{word}_{k, n}\right)
$$

where $K$ is the number of words in the analyzed phrase, $N$ is the number of most similar words identified by word2vec and $\operatorname{int}\left(\operatorname{word}_{k, n}\right)$ denotes the intensity value of $n$ th most similar word to the $k$-th. The intensity value is provided by a particular dictionary (described above).

3. Rule based (rb) W2V search: This feature computing approach is an extension of the previous one with two significant differences:

- It first searches each word in the lists of diminishers, inverters and intensifiers. If the target word is not contained in these lists, we use word $2 \mathrm{vec}$ and the dictionaries to compute the intensity (similarly as in the previous case).

- The obtained value is further fine-tuned using three rules:

- If the phrase contains a diminisher and is positive (intensity $>0.5$ ), it is multiplied by a constant lesser than 1 (if negative, by a constant greater than $1)^{1}$.

- If the phrase contains an intensifier and is positive, it is multiplied by a constant greater than 1 (if negative by constant lesser than 1$)^{1}$.

- If the phrase contains an inverter, its value is subtracted from 1 .

4. Classifier output: We use the 5-level Stanford sentiment classifier proposed by Socher in (Socher et al., 2013). The classifier returns the probabilities of five labels. We compute the final sentiment intensity using a weighted sum of the probabilities with the following weights: $-1,-0.5,0,0.5,1$ ( 1 for very positive phrases). This approach gives a value from the interval from -1 to 1 and it is thus normalized to the interval from 0 to 1 .

\footnotetext{
${ }^{1}$ Constant values were set experimentally on the development data to 0.85 and 1.15 respectively.
}

\section{Other (simple) features}

- Word count: number of words in a phrase

- Diminisher: binary feature, 1 represents a presence of a diminisher (e.g. word "less")

- Inverter: binary feature, 1 represents a presence of an inverter (e.g. word "not")

- Intensifier: binary feature, 1 represents a presence of an intensifier (e.g. word "more") $)^{2}$

\subsection{Regression Model}

To combine the above described features and to obtain the final result we use a regression model. Because of our experience with the Weka package (Hall et al., 2009) we chose this tool for implementation. We did a comparison of several regression models that are implemented in this package and based on the experiments performed on the SemEval development data we chose Gaussian processes (Rasmussen, 2006) as a good candidate for creating the regression model.

\section{Experimental Results}

First, the development data containing 200 annotated words and phrases were provided for each subtask. The general English test dataset contains 2,799 words and phrases and the English Twitter dataset contains 1,069 phrases. All of the presented experiments are done on the general English test dataset. The testing gold data were not provided. Therefore, we have tuned our system on the development data as briefly presented below.

\subsection{Results on General English Test Dataset}

\subsubsection{Performance of the Individual Features}

We first use all proposed features (excluding binary and word count features) separately to check their individual performance. Table 2 shows the results of this experiment.

We have set the parameter $N=16$ in the $W 2 V$ search feature computing approach because this value gave the best results on the development data.

\footnotetext{
${ }^{2}$ The three last features are based on the lists provided by the JRC lexicon with a few words that were present in the development data and not in these lists.
} 


\begin{tabular}{|l|l|l|}
\hline Feature & Kendall & Spearman \\
\hline AFINN direct search & 0.435 & 0.560 \\
JRC direct search & 0.467 & 0.603 \\
SWN direct search & 0.438 & 0.617 \\
\hline AFINN W2V search & 0.510 & 0.698 \\
JRC W2V search & 0.527 & 0.720 \\
SWN W2V search & 0.458 & 0.639 \\
\hline AFINN rb W2V & 0.557 & 0.753 \\
JRC rb W2V & 0.541 & 0.726 \\
SWN rb W2V & 0.464 & 0.650 \\
\hline Classifier output & 0.423 & 0.591 \\
\hline
\end{tabular}

Table 2: Performance of the individual features on general English test dataset

\begin{tabular}{|l|l|l|}
\hline Feature set & Kendall & Spearman \\
\hline without SWN & $\mathbf{0 . 6 5 9}$ & $\mathbf{0 . 8 5 4}$ \\
without binary & 0.630 & 0.831 \\
all features & 0.632 & 0.834 \\
\hline
\end{tabular}

Table 3: Results of the regression model with the different feature sets on general English test dataset

We can conclude that the superior feature is AFINN $r b W 2 V$ followed by $J R C r b W 2 V$ and $J R C$ $W 2 \mathrm{~V}$ search. The other features have somewhat worse results but still might be usable in the final system.

\subsubsection{Results of the Regression Model}

Table 3 presents the results of the final regression model on general English test dataset. This model was trained on the development data. The boldfaced numbers indicate the results submitted to the SemEval. Without SWN means that we have used all the features except the three features based on the SWN dictionary. Without binary means that we have used all the features except the three binary features and the word count feature. Based on this experiment, we have decided not to use the SWN features in the submission because of its slightly lower performance on the development data. We have measured the performance on the development data using a ten fold cross-validation.

\subsubsection{Comparison with the Top Three Teams}

Table 4 shows the final results of the top three teams for the SemEval 2016 task 7 (general English subtask). This table shows that the performance of our system is close to the first one particularly when measured by Spearman correlation.

\begin{tabular}{|l|c|c|}
\hline Team & Kendall & Spearman \\
\hline ECNU & 0.704 & 0.863 \\
UWB (presented) & 0.659 & 0.854 \\
LSIS & 0.350 & 0.508 \\
\hline
\end{tabular}

Table 4: Comparison of the top three teams competing in SemEval 2016 task 7 general English subtask

\begin{tabular}{|l|c|c|}
\hline Team & Kendall & Spearman \\
\hline ECNU & 0.523 & 0.674 \\
LSIS & 0.422 & 0.591 \\
UWB (presented) & 0.414 & 0.578 \\
\hline
\end{tabular}

Table 5: Comparison of the top three teams competing in SemEval 2016 task 7 English Twitter subtask

\subsection{Results on English Twitter}

To show the robustness of our approach, we have evaluated the proposed model also on the English Twitter test data from the SemEval task 7. We did not use any tuning of the method for the rather specific data from Twitter. We just trained the model on the English Twitter development data and tested it on the test data. Table 5 shows the results of three best performing systems submitted to SemEval 2016 task 7, English Twitter subtask. The comparison shows that the performance is not far away from the two better systems.

\section{Conclusion and Perspectives}

We proposed a novel method for sentiment intensity determination. The method uses a rich set of features based on three sentiment lexicons, output of a sentiment classifier and binary features based on lists of modifiers. We first evaluated the features separately on the test data. Then we utilized a Gaussian processes regression model which combines the proposed features and computes the final sentiment intensity. The final system was submitted to the task 7 of the SemEval 2016 competition. The regression model trained on the SemEval development data reached Kendall rank correlation of 0.659 on general English phrases and 0.414 on English Twitter test data.

One perspective is using more sophisticated phrase parametrization. Another one is to prepare word embedding algorithm that groups together words with similar sentiment. 


\section{Acknowledgments}

This work has been partly supported by the project LO1506 of the Czech Ministry of Education, Youth and Sports and by Grant No. SGS-2016-018 Data and Software Engineering for Advanced Applications.

\section{References}

Ramon F Astudillo, Silvio Amir, Wang Ling, Bruno Martins, Mário Silva, Isabel Trancoso, and Rua Alves Redol. 2015. Inesc-id: Sentiment analysis without hand-coded features or liguistic resources using embedding subspaces. SemEval-2015, page 652.

Stefano Baccianella, Andrea Esuli, and Fabrizio Sebastiani. 2010. Sentiwordnet 3.0: An enhanced lexical resource for sentiment analysis and opinion mining. In LREC, volume 10, pages 2200-2204.

Andrea Esuli and Fabrizio Sebastiani. 2006. Sentiwordnet: A publicly available lexical resource for opinion mining. In Proceedings of LREC, volume 6, pages 417-422. Citeseer.

Mark Hall, Eibe Frank, Geoffrey Holmes, Bernhard Pfahringer, Peter Reutemann, and Ian H Witten. 2009. The weka data mining software: an update. ACM SIGKDD explorations newsletter, 11(1):10-18.

Soo-Min Kim and Eduard Hovy. 2004. Determining the sentiment of opinions. In Proceedings of the 20th international conference on Computational Linguistics, page 1367. Association for Computational Linguistics.

Svetlana Kiritchenko, Saif M. Mohammad, and Mohammad Salameh. 2016. Semeval-2016 task 7: Determining sentiment intensity of english and arabic phrases. In Proceedings of the International Workshop on Semantic Evaluation, SemEval '16, San Diego, California, June.

Fangtao Li, Minlie Huang, and Xiaoyan Zhu. 2010. Sentiment analysis with global topics and local dependency. In $A A A I$, volume 10, pages 1371-1376.

Tomas Mikolov, Kai Chen, Greg Corrado, and Jeffrey Dean. 2013. Efficient estimation of word representations in vector space. arXiv preprint arXiv:1301.3781.

F. Å. Nielsen. 2011. Afinn, mar.

Bo Pang and Lillian Lee. 2004. A sentimental education: Sentiment analysis using subjectivity summarization based on minimum cuts. In Proceedings of the 42nd annual meeting on Association for Computational Linguistics, page 271. Association for Computational Linguistics.

Carl Edward Rasmussen. 2006. Gaussian processes for machine learning.
Sara Rosenthal, Preslav Nakov, Svetlana Kiritchenko, Saif M Mohammad, Alan Ritter, and Veselin Stoyanov. 2015. Semeval-2015 task 10: Sentiment analysis in twitter. Proceedings of SemEval-2015.

Richard Socher, Alex Perelygin, Jean Y Wu, Jason Chuang, Christopher D Manning, Andrew Y Ng, and Christopher Potts. 2013. Recursive deep models for semantic compositionality over a sentiment treebank. In Proceedings of the conference on empirical methods in natural language processing (EMNLP), volume 1631, page 1642. Citeseer.

Josef Steinberger, Mohamed Ebrahim, Maud Ehrmann, Ali Hurriyetoglu, Mijail Kabadjov, Polina Lenkova, Ralf Steinberger, Hristo Tanev, Silvia Vázquez, and Vanni Zavarella. 2012. Creating sentiment dictionaries via triangulation. Decision Support Systems, 53(4):689-694.

Xiaolong Wang, Furu Wei, Xiaohua Liu, Ming Zhou, and Ming Zhang. 2011. Topic sentiment analysis in twitter: a graph-based hashtag sentiment classification approach. In Proceedings of the 20th ACM international conference on Information and knowledge management, pages 1031-1040. ACM.

Zhihua Zhang, Guoshun Wu, and Man Lan. 2015. Ecnu: Multi-level sentiment analysis on twitter using traditional linguistic features and word embedding features. SemEval-2015, page 561. 